\title{
UNMANNED AIRCRAFT HAZARDS AND THEIR IMPLICATIONS FOR REGULATION
}

\author{
Kelly J. Hayhurst, Jeffrey M. Maddalon, and Paul S. Miner, NASA Langley Research Center, \\ Hampton, Virginia \\ Michael P. DeWalt and G. Frank McCormick, Certification Services, Inc., Eastsound, Washington
}

\begin{abstract}
Use of unmanned aircraft systems (UASs) has been characterized as the next great step forward in the evolution of civil aviation. Indeed, UASs are in limited civil use in the United States today, and many believe that the time is rapidly approaching when they will move into the commercial marketplace, too. To make this a reality, a number of challenges must be overcome to develop the necessary regulatory framework for assuring safe operation of this special class of aircraft.

This paper discusses some of what must be done to establish that framework. In particular, we examine hazards specific to the design, operation, and flight crew of UASs, and discuss implications of these hazards for existing policy and guidance. Understanding unique characteristics of UASs that pose new hazards is essential to developing a cogent argument, and the corresponding regulatory framework, for safely integrating these aircraft into civil airspace.
\end{abstract}

\section{Introduction}

An unmanned aircraft system comprises an aircraft ${ }^{1}$ flown by a pilot via a ground control station or autonomously through on-board automation, communication links, and any other equipment, including launch and recovery systems, necessary to operate the aircraft safely. In testimony before Congress on July 13, 2006, Nicholas Sabatini, Associate Administrator for Aviation Safety for the Federal Aviation Administration (FAA), stated that "the development and use of unmanned aircraft systems is the next great step forward in the evolution of aviation” [1]. During his testimony, Mr. Sabatini also noted that

\footnotetext{
${ }^{1}$ Unmanned aircraft refers specifically to an air vehicle that does not have an on-board crew. For this paper, we are not considering such aircraft with passengers on board.
}

"development of standards is crucial to moving forward."

Underlying such standards is the notion that UAS operations should not compromise the safety of people-whether on the ground, or in other aircraft. The degree to which existing standards will be appropriate to UASs depends, at least in part, on whether aviation hazards significantly change when the cockpit of the aircraft is separated from the vehicle itself. We assert that fundamental characteristics of UASs and the hazards they present may require a new regulatory framework, rather than adaptation of existing rules.

The next section of this paper notes current trends in the aviation marketplace and advances in technology. These developments may make the "next great step" closer than many people realize, and hasten the need for regulations. In the subsequent sections, a historical perspective is given on hazards with respect to current regulations for manned aircraft. This is followed by a cursory examination of potential hazards that arise from the design and operation of UASs, and a discussion of pitfalls related to applying current regulations to unmanned systems. We conclude by proposing that the unique hazards posed by UAS design, operation, and flight crew invalidate assumptions underlying the current safety assessment process, and thus justify development of a new regulatory framework.

\section{Current Trends}

The number of unmanned aircraft in existence today is astonishing, as is the potential for flying these aircraft for public use and commercial ventures. The following subsections briefly highlight the current state of the UAS market and technologies that enable its rapid growth. 


\section{In the Marketplace}

Use of UASs in military operations is wellknown. As of September 2004, some twenty types of unmanned aircraft, large and small, have flown over 100,000 total flight hours in support of military operations in the Middle East [2]. Worldwide, at least forty-two nations are known to be developing or operating unmanned aircraft [3]. The vast majority are designed for military applications, and most of those are used for surveillance work [4].

This category of aircraft is quite diverse. Vehicles in production range in size from those capable of being hand-launched to vehicles the size of transport aircraft, with weight ranging from a few ounces to over 26,000 pounds, with fixed and rotary wings, and with radically different altitude and endurance capabilities. Nanotechnology may expand this category to include extremely small vehicles, no larger than eight centimeters in length and weighing no more than ten grams [5].

Perhaps less well known is that unmanned aircraft are already in operation in civil or public use applications within the continental United States (US). In the past year, the FAA has granted at least fifty-five Certificates of Authorization (COAs) to government agencies, which permit an agency to operate a particular unmanned aircraft for a particular purpose in a particular area [1]. These authorizations typically include provisions to assure that the unmanned aircraft does not operate in a populated area and that the aircraft is observed, either by a chase aircraft or a trained observer.

One example of operation under a COA comes from the US Customs and Border Protection (CBP) agency. Under its COA, CBP flew a Predator B for over 900 hours between September 2005 and April 2006 to monitor illegal immigrant activities along the US-Mexico border. Temporary flight restrictions were imposed that allowed the Predator $\mathrm{B}$ to fly within a 1,500 square mile area along the border [6]. The surveillance activity has been viewed as a success, and credited with supporting the capture of approximately 1,800 illegal immigrants and the seizure of about 8,200 pounds of illegal drugs [7].

It may not be surprising that UASs are used for border patrol in remote or restricted areas; however, the potential use of UASs in urban areas might be. In June 2006, the Los Angeles County Sheriff's Department made newspaper headlines with their efforts to evaluate replacing manned helicopters with a portable unmanned drone for tasks such as searching for lost hikers or missing children, surveying fire zones, and chasing suspects fleeing on foot [8]. Current regulations require that operators of unmanned aircraft, for purposes other than recreation, must comply with FAA guidance and apply for a COA [9]. While the FAA has not issued a COA in this particular case, the Los Angeles County Sheriff's Department exemplifies an eager civil market for unmanned aircraft.

The commercial market is growing globally as well. Japan is often cited as having the most successful commercial operation of unmanned aircraft. In 1958, Japan began using manned helicopters to spray agrochemicals for insect and disease prevention in rice fields. Unmanned helicopters joined the effort in 1991. Today, more than 2,000 unmanned industrial helicopters are used in Japan, primarily in the agricultural sector [10, 11]. Numerous other possibilities exist for commercial applications, including communications, commercial security surveillance, aerial advertising, news and media support, and air freight, to name only a few.

\section{Current Trends in Technology}

Advances in automation technology have been at least one key to the rapid evolution of unmanned aircraft. Similarly, automated systems are the foundation of many visions to make air travel safer, more secure, more dependable, and available to more people than it is today [12]. The airspace system already accommodates some degree of autonomous operation at the aircraft level, as described by Hadden [13]:

Except during take-off and the final stages of landing, the modern commercial aircraft is routinely being flown by computers, monitored by human pilots. The systems in the latest generation of commercial aircraft commonly have fault monitoring and diagnostic functions which can cope with many failure conditions without pilot intervention. Automatic landing including flare and ground roll has been commonplace 
for many years. When automation of the take-off segment of flight also becomes common it may be the norm for airliners to complete their missions without operation of the primary flying controls by a human pilot at any stage.

The trend toward increased reliance on automation is evident in air traffic control as well. Plans for the Next Generation Air Transportation System envision using 4-dimensional trajectories (time-based paths from beginning to end) as the basis for planning and executing system operations. Automation will continuously analyze trajectories, taking into account weather information and forecast uncertainties, to support safe separation and efficient traffic flow [12].

To date, regulation has adjusted to accommodate increased automation. Some have suggested that modifications to existing rules can accommodate UASs [2, 13]. For example, the US military's roadmap for unmanned aircraft calls for interpreting the existing Title 14 Code of Federal Regulations (CFR), known as Federal Aviation Regulations or FARs, "to also cover unmanned aviation and avoid the creation of dedicated UA regulations as much as possible" [2]. It is not clear, however, whether existing regulations that are based on a historical pairing of pilot and plane can be adapted to accommodate UASs, or whether UASs constitute a fundamentally different category of aircraft requiring their own set of regulations.

To determine whether change to existing regulations is tractable, it is important to understand the underpinnings of those regulations. Those underpinnings include hazards and their role in the safety assessment process.

\section{Hazard Analysis Today}

In civil aviation, the pursuit of safety inevitably focuses attention on hazards. Aircraft designers, flight crews, mechanics, and regulators traditionally devote a great deal of effort, both explicitly and implicitly, to the identification and mitigation of aviation's hazards. For the purpose of this discussion, we use the term "hazard" loosely to describe any situation or circumstance that may lead to danger or risk. Our concerns with hazards are those associated with UAS design, with UAS operation, and with the provision of air traffic services to UASs.

In some cases, hazard analysis begins with reasoning about aircraft-level failure conditions or hazards-loss of pitch control, loss of all hydraulics, misleading attitude display—and works backward through the events, conditions, and circumstances that might create those hazards. In other cases, the reasoning begins with assumptions about undesirable events, such as engine-rotor burst or bird strikes, primarily in an attempt to identify and characterize potential threats to redundancy and independence of systems. Other analyses support and complete the safety-assessment picture. Taken together, these analyses provide a comprehensive understanding of operations and vulnerabilities at both a system level and an aircraft level.

Where necessary or desirable to do so, hazards are mitigated through changes to aircraft design, operational procedures, training, inspection, or maintenance. The changes themselves must then be confirmed as acceptable.

For manned aircraft, these processes are understood and practiced more or less universally. But there is a hidden assumption in this understanding, an assumption thought to be selfevident and rarely acknowledged. The assumption is worth stating here explicitly: aviation's conventional hazards are hazards because they place human passengers and crew at risk. Implicit in most safety-related regulations is a desire to limit or eliminate harm to passengers and crew aboard aircraft, with a secondary consequence of limiting harm to people on the ground. Other consequences, notably economic consequences, are unrelated to the primary intent.

In any proposed new rule or compliance method, regulators and designers are, in effect, treating all considerations as proxies for risk to the passengers aboard an aircraft. For instance, substantial engineering resources are spent evaluating the "crew workload" associated with new systems and procedures. Increased workload is bad, not because we are worried about crew exertion and toil per se, but because we know that an overtaxed, confused, and distracted pilot is more likely to be part of an accident that injures or kills people aboard the aircraft. 
Many existing rules designed to protect passengers and crew may be irrelevant to UASs. Conversely, rules needed for the safe operation of UASs may sometimes be absent from the current regulations that govern manned flight. Regulation of unmanned systems must confront this mismatch directly.

\section{Hazard Analysis for UAS}

Understanding the hazards posed by an aircraft is an essential part of determining what regulations are needed to ensure safety. For unmanned aircraft, much of the hazard analysis to date [13-16] has focused on high-level concerns: ground impact with collateral damage to people and property, and midair collisions with other manned aircraft. The analysis often is predicated on the notion that the capability of an aircraft to cause harm to third parties is roughly proportional to its kinetic energy on impact. The analysis typically uses the kinetic energy and population densities to create a risk metric that is compared with historical accident data from manned aircraft.

This top-down approach to hazard analysis provides a valuable frame of reference for establishing broad safety objectives. However, this analysis implicitly assumes that hazards for unmanned aircraft are essentially the same as hazards for manned aircraft. To determine whether UASs introduce new hazards, we consider the question: do aviation hazards significantly change when the cockpit of the aircraft is separated from the vehicle itself?

In the next section, we look at the recent crash of a Predator B to provide some insight, and then discuss some of the hazards that are highlighted by this accident.

\section{An Example UAS Accident}

In the early morning hours of April 25, 2006, a Predator B, providing surveillance of the USMexico border, went out of control and subsequently crashed on public property near Nogales, Arizona. According to the preliminary report from the National Transportation Safety Board (NTSB) [17], the ground control station for the flight included two control consoles, designated pilot payload operator (PPO)-1 and PPO-2, with identical aircraft controls. For a typical flight, a certified pilot uses PPO- 1 for flight control, while a $\mathrm{CPB}$ agent controls the surveillance camera from PPO-2. The NTSB reported that the following events happened on April 25:

The pilot reported that during the flight the console at PPO-1 "locked up", prompting him to switch control of the UAV [unmanned aerial vehicle] to PPO-2. Checklist procedures state that prior to switching operational control between the two consoles, the pilot must match the control positions on the new console to those on the console, which had been controlling the UAV. The pilot stated in an interview that he failed to do this. The result was that the stop/feather control in PPO-2 was in the fuel cutoff position when the switch over from PPO-1 to PPO-2 occurred. As a result, the fuel was cut off to the UAV when control was transferred to PPO-2.

The pilot stated that after the switch to the other console, he noticed the UAV was not maintaining altitude but did not know why. As a result he decided to shut down the [ground control station] so that the UAV would enter its lost link procedure, which called for the UAV to climb to 15,000 feet above mean sea level and to fly a predetermined course until contact could be established. With no engine power, the UAV continued to descend below line-of-site communications and further attempts to reestablish contact with the UAV were not successful.

This example raises several issues relevant to UAS hazard analysis, especially with respect to flight control. The first and perhaps most obvious issue is that many flight-critical control functions for a UAS will be located within the ground control station, not on the aircraft. So, proper design and use of flight controls is essential, regardless of where those controls reside. In this example, the secondary control console was able to command an unsafe fuel state to the shutoff valve. While procedures were in place to avoid this, there was no automation or other means similar to back-driven controls in manned aircraft which would ensure synchronization between control consoles.

Another issue concerns the coupling of flight control and payload control. The hazards 
associated with the payload and the mission are not necessarily at the same level of criticality as the control of the aircraft. Wherever there are shared resources or interactions between the payload and the air vehicle, there is a need to analyze and manage this sharing of resources to ensure that all hazards have been mitigated.

A third issue worth noting involves the tradeoff between manual control and autonomous operation. In manned aircraft, the pilot is always responsible for monitoring the autonomous operation of the aircraft and can intervene to mitigate most operational hazards. The loss of a command and control link in a UAS, however, removes the possibility of human intervention. In the Predator accident, the amount and type of control authority available to the aircraft after loss of link was dependent on inadequate assumptions about the state of the aircraft (for example, the ability to control thrust).

\section{Discussion of UAS Hazards}

In this section, we broaden the discussion of hazards to three domains:

UAS Design Domain: includes all components, parts, and elements of an unmanned system. This includes the air vehicle, control stations, communication links, and any specialized launch and recovery equipment, and payload.

UAS Flight Crew Domain: includes the capabilities, human factors, and workload of the human pilot of the vehicle and operator of the payload.

UAS Operational Domain: includes the vehicle's operations within both controlled and uncontrolled airspace, above both populated and unpopulated areas, over either land or water. This includes take off, landing, and any airport operations.

While the domains are unique, decisions within one domain have a profound effect on the design and hazards in other domains. The discussion includes some of these interactions as examples; however, the examples are not intended to be a comprehensive study of all UAS hazards.

For each domain, important differences between manned and unmanned systems are identified first. Then examples are given that describe unique hazards introduced by UASs or known hazards that take on a new spin. It should be noted that not all of these hazards apply to all UASs; they are included because the UAS design or operation space might be unduly restricted if the regulations do not account for them.

\section{Design Domain}

The design domain for manned aircraft systems comprises all of the elements that are part of the air vehicle itself. For a UAS, this domain extends beyond the vehicle to include any external systems that participate in control of the aircraft. The vehicle, the control station with pilot displays and flight controls, the communication links for command and control, any special launch and recovery systems, and even the payload are all part of the UAS design domain.

Communication links are critical to UAS operations. This includes links between the vehicle and the control station and also between the pilot and ATC. There are typically three types of radio communications links between the vehicle and the control station: a flight control link; a telemetry link for transmitting vehicle health, status, and situation awareness data; and, a link used to control, manage, or monitor payload sensors or equipment [4].

UAS pilots may communicate with ATC in the same way that conventional pilots do, using voice radio frequencies. But, UAS pilots also have additional options, such as telephone connections, between the ground station and ATC. Other possibilities include using the unmanned aircraft as a relay between the UAS pilot and ATC, or (perhaps in the future) for the air vehicle to automatically respond to ATC commands.

While the cockpit of a manned aircraft provides all of the equipment necessary for flight control, the UAS control station provides the facilities necessary for remote control of the air vehicle and payload. The control station may include an operator console, with command-input device (joysticks and switches), video monitors, control station data encoder and transmitter, data and video receivers and antennas. Size and equipage of the control station varies. Location varies, too. A control station may be in one fixed 
location, or may be mobile. Many military ground control stations look like specially outfitted trailers, but a control station might also look like a conventional computer placed in an officeperhaps far away from an airport. In addition, the control station may not necessarily be located on the ground. For example, the control station could be in an aircraft or on a ship.

A final difference worth noting involves the payload. Instead of transporting people, each UAS typically carries payload related to an operational mission. Design of commercial manned aircraft separates the effects of payload from vehicle control. For unmanned vehicles, size and weight constraints may motivate system designs that integrate payload and flight control. This is especially true for small UASs. In other cases, the nature of the mission may dictate integration, such as surveillance systems that automatically track their targets without pilot input.

The communication links, control station, and payload have the potential to introduce new hazards. From a system design perspective, loss of vehicle control is a primary concern. Below are brief descriptions of potential problems that may lead to loss of vehicle control.

\section{Loss of the command and control link}

In manned aircraft—absent failures-pilots always have command of their aircraft. However, in a UAS, the command and control link may be lost, leaving the UAS pilot without command of his aircraft. Radio links are known to be vulnerable to interruptions, latency, and data degradation. So, intermittent loss of a flight control link is an expected event, not a failure. As such, UAS designs provide means to deal with these interruptions. Those means, however, can provide only temporary safety measures, at best. Persistent loss of link can lead to complete loss of vehicle control.

Because the command and control link is part of a flight critical function, much greater attention must be paid to its integrity. Having a secure civil frequency for command and control, within the FAA's protected spectrum, is a major challenge for UAS [4]. The pilot must be confident that the information presented on the displays is from the vehicle under control (that is, that the information is not for some other vehicle, nor is it from a malicious source) and the communication uplink to the vehicle must be protected from interference (both spurious and malicious) ${ }^{2}$.

\section{Reliance on third-party communication}

Manned aircraft typically rely on communication and navigation systems provided and evaluated by the regulatory authorities rather than relying on third-party communication systems. For UAS, where the control station and the aircraft may be separated by hundreds or thousands of miles, there may be a greater dependence on third-party communication systems for flight critical functions. Issues related to the reliability and availability of third party communication systems may need to be addressed.

\section{Ground station environment}

Some hazards associated with traditional cockpit environments, such as smoke, fire, and toxic fumes and flammability of materials, will have to be considered for ground stations, too. Other hazards that directly impact a control station and UAS pilot, may not directly impact the vehicle. For example, events such as earthquakes or tornados may impact the control station, but not impact the aircraft in flight. Although hazards in the ground station environment differ from the traditional cockpit, they may be comparable to some hazards in the physical environment of ATC and may be dealt with accordingly.

Security of the control station is another matter. Because the control station may be located outside the relatively secure confines of a military base or an airport, additional security measures may be required. An aircraft that is controlled by a malicious person is even more dangerous than an uncontrolled aircraft. Analysis of the control station hazards should take into account impact on the ability to maintain control of the vehicle.

\section{Shared resources}

The UAS must be evaluated with respect to potential interactions between the vehicle and its

\footnotetext{
${ }^{2}$ It is important to recognize that solutions to this problem developed for military systems may or may not be adequate for private use. For example, military systems may have bandwidth that is unavailable to commercial UAS operators.
} 
payload. When resources are shared between flight functions and payload functions, proper management of these shared resources is essential. For example, the payload and the flight critical systems may share power and cooling systems; therefore, it must be assured that the payload's power and cooling needs do not interfere with flight critical functions.

\section{Payload induced loss of vehicle control}

While commercial manned aircraft separate the effects of payload from vehicle control, some UAS designs may require their integration. For example, the ability for payloads to track a target, such as a thermal signature, may require direct control inputs to the aircraft's avionics.

In contrast to these new hazards, there may also be areas in which UAS design may reduce certain hazards. Two of these are discussed below.

\section{Payload mitigation of certain failures}

A sophisticated payload may provide a means to mitigate certain failures. For instance, a surveillance instrument (for example, radar, forward-looking infrared, or camera) may be used to provide sensor data, in essence providing a backup for primary sensors. A payload that consists of communication equipment may provide a backup for loss of the command and control link.

Significant questions must be addressed, however, about whether payload generated information should be used in support of flight-critical functions.

\section{Alternate communications with air traffic control}

Loss of communication for manned aircraft during a flight under Instrument Flight Rules requires strict lost communication procedures to be followed by both the on-board pilot and the air traffic controllers (Federal Regulations 14 CFR, Part 91.185). This problem may be mitigated for unmanned aircraft because there are alternate communication means available (for example, telephone lines). This presumes that regulations would be in place to require this type of back up communication both at the UAS crew stations and at ATC centers. The UAS flight crews would have to be trained and certified to properly use this facility, but it should result in considerably less disruption than loss of communication in manned aircraft.

\section{Flight Crew Domain}

Most UASs provide some method of displaying the state of the air vehicle to the pilot and a means for the pilot to change this state. In this section, the term pilot is used for the person in direct control of the UAS. UAS crew includes the pilot and any operators of mission related equipment aboard the aircraft.

The separation of the crew environment from the aircraft introduces a number of new hazards and training requirements that are not covered by the current training and certification requirements for manned vehicles. One such difference in training relates to physical cues. Pilots of manned aircraft require training to properly interpret the physical sensations induced by the movement of the aircraft. For example, training in recognition and recovery from vertigo is required for all pilots during instrument training, which presumably will be unnecessary for a UAS crew.

Also, the UAS crew members may be expected to control more than one air vehicle at a time. If there are multiple UAS crew members for a given air vehicle, the specific cockpit resource management (CRM) procedures in manned aircraft would not necessarily be correct for UAS crews. For example, some current CRM techniques require one pilot to be assigned visual acquisition duties while the other pilot is responsible for monitoring the flight displays. The exact type of training and controls will depend heavily on the type of certification requirements and categories of UASs. However there are some hazards unique to all UASs that will have to be addressed.

Many of the hazard definitions in the advisory material for safety (such as FAA Advisory Circular 23.1309-1C [18]), take into account increases in pilot and flight crew workload. In those definitions, workload is defined based on a traditional cockpit environment where crew replacements and rotations are not possible. Such definitions will be inadequate for UAS crew workload where the degree of automation on board the air vehicle will be a significant factor. 


\section{Reduced pilot situational awareness}

A pilot aboard an aircraft can use physical senses such as sight, smell, and touch to detect certain hazardous situations. Removing these "sensors" from the aircraft will have an unknown effect on the ability to detect some hazardous situations such as equipment failures and bad weather. The issues associated with developing sensors to replace this functionality and determining the most effective way to present this information to the UAS pilot are largely unknown. At best the introduction of a communication link will add delay. The degree to which this additional delay affects the pilot's performance is unknown.

Situational awareness is key to the ability to mitigate problems. In traditional manned designs, the on-board pilot plays an important role in systems monitoring and risk mitigation. The situational awareness of a UAS pilot is at least different, if not reduced, compared with an onboard pilot. As such, effectiveness of a UAS pilot to monitor systems and mitigate risk is unknown. The potential exists to have on-board automation perform some of these functions; however, this is placing automation in a new role. If this role is shared between pilot and automation, then the blending of automation and UAS crew inputs will have to be considered. This is complicated further because the on-board automation may have a more accurate view of the state of the vehicle.

\section{Multiple vehicle control}

One possibility for ground control stations is the ability for a single pilot to control multiple vehicles. This feature is being actively pursued by the developers of military UASs. In this situation, new hazards arise from both an increased pilot workload and ensuring that the information presentation does not confuse the pilot about which aircraft is being controlled. Training specific to controlling multiple aircraft may be required.

\section{Equipment failure training}

In any aircraft system, there are hazards introduced by equipment failures. An on-board flight crew may be able to physically or visually establish the extent and type of some failures such as fire, smoke, and vibration, and they may have direct means to mitigate some of those failures. For example, a crew member may be able to set a fuel cutoff valve in a Part 23 aircraft. Equipment failures on the vehicle may be dealt with through design mitigations, but also through UAS flight crew training and procedures specific to remote operation.

Training for environmental hazards on the ground

As mentioned above, ground control stations are subject to physical environmental hazards such as a fire, and to other disturbances such as an earthquakes or tornados. A UAS flight crew may require training relative to establishing a safe control state of the air vehicle in the event of such hazards. While such hazards will have to be dealt with by design mitigations, procedural mitigations also may be necessary.

\section{Operational Domain}

Civil airspace contains numerous aircraft which have been allocated both static (Restricted) airspace and dynamic airspace (protective separation boundaries between aircraft assigned by ATC). Some of the characteristics of unmanned aircraft may present challenges to the rules for airspace use and provision of air traffic services. Chief among these challenges is the capability to "see and avoid" ${ }^{3}$ that would enable a UAS to detect air traffic, hazardous weather, terrain, and other obstructions and safely maneuver around them as per FAR Part 91.113 [4].

Other challenges relate to operational flexibilities inherent in unmanned aircraft. The scope of UAS missions varies widely. Some missions may require transit from one location to another, while other missions may require loitering over a particular location for an extended time. Some UASs may operate for substantially longer periods of time or at higher altitudes than their manned counterparts.

UASs have significantly more options for surface operations, including pre-flight preparation, take-off or launch, and landing than manned aircraft that are typically dependent on services provided by conventional airports. Launch and recovery systems common in military aircraft, but deemed too risky for civil operation, may be used for UASs. For

\footnotetext{
${ }^{3}$ Also referred to as "sense and avoid” or "detect and avoid”.
} 
instance, launch systems may include catapult or rocket assisted takeoffs. These systems may be fixed at an airport or part of a mobile launch system. Recovery systems may include arresting wires, net capture systems, and recovery parachutes. These, too, may be located at an airport or as part of a mobile recovery system.

In emergency situations, UASs may have options that are unavailable to manned aircraft including pyrotechnic destruction systems and a dedicated structure at an airport to contain the crash (a "crashport"). These systems can be used to maintain safety of people and property but result in a vehicle hull loss.

Many of the operational hazards for UAS are associated with the loss of the command capability and subsequent loss of control of the aircraft as described in the design domain. The following discussion focuses on additional hazards relevant to UAS operations.

\section{Situational awareness for ground operations}

Ground operations for UASs vary extensively. In some cases, the UAS pilot may be responsible for pre-flight procedures, start, and take-off or launch. In other cases, the UAS pilot may be far away from the vehicle and rely on ground support crew for some or all of those functions. In the latter case, the UAS pilot may be unaware of ground personnel or obstacles around the vehicle. The pilot may start the engine or cause the vehicle to make a sudden move that ground personnel were not expecting.

\section{Safety margins for ground operations}

In general, safety margins with respect to separation standards are much smaller on the ground than in the air. For example, most aircraft controlled by ATC are separated by several miles in flight. In a conventional airport setting, however, aircraft are separated by only a few tens of feet on a taxiway. If unmanned aircraft are operated in conventional airport settings, existing standards for safety margins between aircraft on the ground may be insufficient given the delay inherent between a UAS pilot's command and subsequent vehicle movement.

\section{Entrance to controlled airspace}

In most cases, manned aircraft enter controlled airspace through relatively few areas such as airports and helipads. UAS could enter controlled airspace through these means or, may also enter through uncontrolled airspace. UAS have a much larger range of options for launch and recovery (catapults, hand launch, bungee cords), so they could enter uncontrolled airspace from almost anywhere (field, rooftop, moving truck, ship, etc.) $[2,20]$, then transition to controlled airspace.

Although some manned aircraft enter controlled airspace through uncontrolled airspace, for certain UAS applications, this may be their normal mode of operation. Where and how UAS enter controlled airspace may impact air traffic management. As such, this may change operational, training, or equipage requirements.

\section{Flight termination}

One of the unique capabilities of a UAS from a safety standpoint is the ability to terminate flight prematurely without concern for passengers. However, such termination may injure people on the ground. Simply terminating the flight when something goes wrong is unacceptable. To ensure that people on the ground are not affected, termination of flight should place the vehicle in an unpopulated area.

This may not be enough, however, to ensure the safety of people on the ground. If the vehicle is carrying dangerous materials, then terminating the flight may endanger people on the ground - even if they are not directly affected by the impact. For example, an aircraft with a large quantity of fuel could start a forest fire.

\section{Additional air traffic control workload}

If the communication link between the aircraft and UAS pilot is lost or corrupted, ATC may have a better view of the vehicle than the UAS pilot. Consequently, ATC may have a new role in reporting the state of the vehicle to the UAS pilot in certain types of failure situations. Such a role would impose additional workload on ATC. 


\section{Implications for Regulation}

The ultimate acceptance of unmanned aircraft in civil airspace critically depends on maintaining the perceived level of safety of the current airspace environment $[2,16,19]$. The proposed baseline is that UASs establish an equivalent level of safety (ELOS) to that of corresponding manned aircraft operations. Any regulatory framework that attempts to establish an ELOS must recognize the different types of operations and architectures to be used for UASs and the unique hazards they present.

\section{Regulation of UAS design, operation, and flight crew}

Current regulations for certifying and operating air vehicles can be categorized as follows: vehicle design requirements, operational requirements, continued airworthiness requirements, and flight crew certification requirements. The regulatory requirements that govern the acceptability of aircraft design, operations, and pilot requirements can be found in 14 CFR, Chapter I. The design of aircraft (14 CFR Parts 23, 25, 27, 29, 31, and 33) relate to requirements for specific aircraft types and propulsion mechanisms with certain characteristics. Those regulations impose various requirements on the design and operation of the aircraft and on flight-crew acceptance that would not necessarily be required for a given UAS.

For example, hazards resulting in airframe destruction are, by definition, considered catastrophic for manned aircraft, due to the direct effects on passengers and crew. From a publicsafety standpoint, the destructive sacrifice of a vehicle may be perfectly acceptable if that sacrifice avoids an undesirable collision with a manned aircraft or people or property on the ground. In short, new regulations are needed to deal with the unique hazards, such as loss of the command and control link and loss of situational awareness, associated with UASs.

It also may be necessary to identify new types of operations for UASs. Traditional distinctions among commercial, private, and recreational operations will not have the same meaning for UASs. In general, loss of control of a unmanned aircraft has no analog to manned aircraft, so new operational rules will have to address loss of control differently. This may involve airspace restrictions, operational mitigations for certain failure conditions such as loss of the communication link, and so on. In many cases, such as the loss of communication link, new operational requirements may have to be implemented as part of aircraft design.

Substantially different regulations for UAS flight crew may be needed, too. All of the current regulations governing pilot qualifications (14 CFR Chapter I, Subchapter D), certification, and training are based on the implicit assumption that the pilot is collocated with the vehicle being controlled. The current regulations that deal with certification of pilots provide for different types of certificates (such as private, commercial, and instructor), category ratings (such as single engine land and multiengine sea), operational ratings (such as Instrument Flight Rules and Visual Flight Rules), and type ratings for specific aircraft (such as a Boeing 737 or Airbus A320). In all of the cases, when the operating characteristics of the aircraft are significantly different from existing aircraft, additional regulations are created to ensure the qualification of the flight crew.

The inherent separation of the control station from the air vehicle provides a much greater disparity than current aircraft, that may require different rules for category, operational, and type ratings. Under the current policy [9], UAS crew members need only meet minimum medical requirements, have passed the private pilot knowledge test, and, if flying on an instrument flight plan, be a certified pilot with an instrument rating. None of these regulations deal with establishing qualifications for handling the hazards identified in the previous sections. The large variety of UASs in terms of size, autonomy level, and operational use, makes developing certification criteria for human factors extremely difficult [4]. Decisions affecting the architecture of the different domains as well as the interaction among these domains will need to influence the regulations relating to operator training, certificate types, and operator privileges.

\section{Regulatory Challenges}

The hazards in this paper have been grouped according to specified domains that map to the basic structure of the regulations. The UAS design 
domain would be addressed in 14 CFR Subchapter $\mathrm{C}$, the flight crew domain in Subchapter D, and the operational domain in Subchapters E, F and G. The challenge is to establish regulations unique to UAS hazards that permit addressing hazards at the service level and permit optimization of design parameters to take advantage of the unique characteristics of UASs. For example, redundancy and autonomy (such as preprogrammed flight plans) could be traded against the integrity of ground monitoring (electronic or visual) and safetymitigation systems aboard the aircraft (detonators, parachutes).

The causes of the Predator accident discussed earlier in the paper are clearly identifiable and preventable. Prevention of such accidents requires that current regulations be augmented with rules for UAS operation, design of UAS control consoles, UAS communication links, acceptable levels of UAS autonomy, training of UAS crews, and so on. Existing regulations fail to address these and many related areas.

\section{Summary}

The use of unmanned aircraft in national airspace has been characterized as the next great step forward in the evolution of civil aviation. Unique characteristics of unmanned aircraft allow a wide range of applications ill-suited for manned systems. The tremendous economic potential is obvious, providing substantial motivation for taking that step. Regulation is crucial to moving forward.

The unique characteristics of UASs that provide such promise, also create regulatory challenges. Even in the most conventional UAS designs, significant new hazards arise from ground control stations, communication links, and specialized avionics. The effect of failures in these elements brings a new twist to long-established hazards such as loss of aircraft control and loss of situational awareness. In this paper, we have provided a cursory look at some of those twists and related deficiencies in existing regulation for UAS design, operation, and flight crew.

Allowing routine and safe access of UASs to civil airspace is clearly a complex problem that involves numerous issues including regulation. Adaptation of existing rules, however, may be inadequate to handle the new hazards posed by UASs. This leads us to the proposition that UASs are a fundamentally new type of aircraft that will require a new regulatory framework to both maintain the safety of the national airspace system and to enable the full benefit of unmanned aviation.

\section{Acknowledgements}

We would like to thank Michael Holloway and Brent Weathered of NASA Langley Research Center for extensive comments and suggestions that substantially improved the quality of this paper. This work was funded in part by NASA Langley Research Center under contract number NAS102117.

\section{References}

[1] Sabatini, Nicholas A., 13 July 2006, Statement of Nicholas A. Sabatini, Associate Administrator for Aviation Safety, Before the Committee on Commerce, Science, and Transportation, on Unmanned Aircraft Systems in Alaska and the Pacific Region: A Framework for the Nation.

[2] Office of the Secretary of Defense, August 2005, Unmanned Aircraft Systems Roadmap, 20052030.

[3] van Blyenburgh, Peter, 9 March 2006, UAV Systems: Global Review, Unmanned Vehicle Systems (UVS) International, Avionics '06 Conference, Amsterdam, The Netherlands.

[4] DeGarmo, Matthew T., November 2004, Issues Concerning Integration of Unmanned Aerial Vehicles in Civil Airspace, MP 04W0000323, The MITRE Corporation.

[5] McKaughan, Jeff, 19 February 2006, Nano Nano, Special Operations Technology Online Edition, Volume 4, Issue 1, access at http://www.special-operationstechnology.com/article.cfm?DocID=1342

[6] Kostelnik, Major General Michael, 29 March 2006, Statement of Major General Michael Kostelnik, Assistant Commissioner for the Bureau of Customs and Border Protection Office of Air and Marine, Before the Committee on Transportation and Infrastructure, Subcommittee on Aviation, on 
Unmanned Aerial Vehicles and the National Airspace System.

[7] McCombs, Brady, 26 April 2006, Borderwatching drone crashes after blackout; cause unknown, Arizona Daily Star.

[8] Wood, Daniel B., 22 July 2006, It's a kite. It's a model airplane. It's ... the sheriff!, The Christian Science Monitor, access at http://www.csmonitor.com/2006/0711/p01s01usju.html

[9] McGraw, J. W., September 2005, Unmanned Aircraft Systems Operations in the U.S. National Airspace System-Interim Operational Approval Guidance, AFS-400 UAS Policy 05-01, Dept. of Transportation, Federal Aviation Administration, Washington, DC.

[10] Sato, Akira, June 2002, Civil UAV Applications in Japan and Related Safety \& Certification, UAV 2002 Conference \& Exhibition, Paris, France.

[11] Enderle, B., 20-23 May 2002, Commercial Applications of UAV's in Japanese Agriculture, AIAA-2002-3400, 1st UAV Conference, Portsmouth, Virginia.

[12] US Joint Planning and Development Office, 2005, 2005 Progress Report To The Next Generation Air Transportation System Integrated Plan, (to be published), access at http://www.jpdo.aero/pdf/ngats-np_progress-report2005.pdf

[13] Weibel, Roland E., R. John Hansman, Jr., 2022 September 2004, Safety Considerations for Operation of Different Classes of UAVs in the NAS, AIAA-2004-6244, AIAA's 4th Aviation Technology, Integration and Operations (ATIO) Forum, Chicago, Illinois.

[14] Weibel, Roland E., R. John Hansman, Jr., 2629 September 2005, An Integrated Approach to Evaluating Risk Mitigation Measures for UAV Operational Concepts in the NAS, AIAA-20056957, Infotech@Aerospace, Arlington, Virginia.

[15] Haddon, D. R., C. J. Whittaker, August 2002, Aircraft Airworthiness Certification Standards for Civil UAVs. Civil Aviation Authority, UK.

[16] Clothier, Reece, Rodney Walker, 2006, Determination and Evaluation of UAV Safety
Objectives, 21st International Unmanned Air Vehicle Systems Conference, Bristol, United Kingdom, pages 18.1-18.16.

[17] National Transportation Safety Board, Identification: CHI06MA121, 14 CFR Public Use Incident occurred Tuesday, April 25, 2006 in Nogales, AZ.

[18] Gallagher, Michael, 12 March 1999, Advisory Circular AC No. 23.1309-1C, Equipment, Systems, and Installations in Part 23 Airplanes, Dept. of Transportation, Federal Aviation Administration.

[19] Civil Aviation Authority, United Kingdom, 12 November 2004, CAP 722 - Guidance for Unmanned Aerial Vehicle (UAV) Operations in UK Airspace.

[20] Department of the United States Army, April 2006, Army Unmanned Aircraft System Operations, FMI 3-04.155. 